\title{
Safety-Liveness Exclusion in Distributed Computing
}

\author{
Victor Bushkov \\ EPFL, IC, LPD \\ victor.bushkov@epfl.ch
}

\author{
Rachid Guerraoui \\ EPFL, IC, LPD \\ rachid.guerraoui@epfl.ch
}

\begin{abstract}
The history of distributed computing is full of trade-offs between safety and liveness. For instance, one of the most celebrated results in the field, namely the impossibility of consensus in an asynchronous system basically says that we cannot devise an algorithm that deterministically ensures consensus agreement and validity (i.e., safety) on the one hand, and consensus wait-freedom (i.e., liveness) on the other hand.

The motivation of this work is to study the extent to which safety and liveness properties inherently exclude each other. More specifically, we ask, given any safety property $S$, whether we can determine the strongest (resp. weakest) liveness property that can (resp. cannot) be achieved with $S$. We show that, maybe surprisingly, the answers to these safety-liveness exclusion questions are in general negative. This has several ramifications in various distributed computing contexts. In the context of consensus for example, this means that it is impossible to determine the strongest (resp. the weakest) liveness property that can (resp. cannot) be ensured with linearizability.

However, we present a way to circumvent these impossibilities and answer positively the safetyliveness question by considering a restricted form of liveness. We consider a definition that gathers generalized forms of obstruction-freedom and lock-freedom while enabling to determine the strongest (resp. weakest) liveness property that can (resp. cannot) be implemented in the context of consensus and transactional memory.
\end{abstract}




\section{Introduction}

The correctness of a distributed algorithm is expressed through safety and liveness properties [30, 1, 33, 29]. These properties can be defined as sets of histories (traces). More specifically, a safety property is defined as a prefix-closed and limit-closed set of well-formed histories [29]. Whereas, a liveness property is defined as a set of histories that permits any finite well-formed history, i.e., for every finite history there exists a continuation of that history in the liveness property [29].

Because a liveness property basically states that certain 'good' events should eventually happen while a safety property states that certain 'bad' events should never happen, it occurs sometimes that a safety property makes it impossible to guarantee a liveness property, i.e., when the 'bad' and the 'good' coincide. For example, it is impossible to implement a consensus shared object using only asynchronous read/write shared memory while ensuring both wait-freedom, a liveness property, and agreement and validity, a safety property $[8,28]$. The history of distributed computing is full of such trade-offs $[7,19,37,22,13,14,6,12,4]$.

We ask the questions of safety-liveness exclusions: namely if we can determine, given any safety property $S$, the strongest (resp. weakest) liveness property that can (resp. cannot) be achieved with $S$. In the context of consensus for instance, this would mean determining the strongest liveness property that can be ensured while still ensuring consensus safety. Given that consensus is at the heart of state machine replication [26, $32,11,27]$, addressing such questions could enable us to determine the maximum availability (liveness) to be expected if the consistency (safety) of a shared distributed service needs to be preserved. More specifically, given $\mathbb{S}$ and $\mathbb{L}$ the sets of safety and liveness properties respectively, we seek to determine if there is a mapping $f_{w}: \mathbb{S} \rightarrow \mathbb{L}$ (resp. $f_{s}: \mathbb{S} \rightarrow \mathbb{L}$ ) that maps every safety property $S \in \mathbb{S}$ onto the weakest (resp. strongest) liveness property $L \in \mathbb{L}$ that cannot (resp. can) be achieved with $S$.

When the strongest possible liveness property in a given context is known to be implementable with a given safety property, the answer to the above question is trivial. In many cases, however, the question is more challenging. We show in this paper that, maybe surprisingly, in the general case, it is impossible to determine the strongest (resp. weakest) liveness property that can (resp. cannot) be achieved with a given (non-trivial) safety property. In other words, the safety-liveness exclusion mappings do not exist.

We proceed as follows. We consider a general asynchronous shared-memory distributed system, in which any number of processes can fail by crashing, and we say that a liveness property excludes a safety property of a shared object if every implementation of that object which ensures the safety property, violates the liveness property. We then show that there is no strongest liveness property that does not exclude a given safety property in a non-trivial case, and give a necessary and sufficient condition when there is a weakest liveness property that excludes a given safety property.

- If $L_{\max }$ is the strongest possible liveness property among all liveness properties ( $L_{\max }$ is a liveness property that requires progress for all non-crashed processes), then the first result states the following. If there exists a strongest liveness property that does not exclude a given safety property, then such property must be $L_{\max }$.

- The fact that a liveness property $L$ excludes a safety property $S$ means that there is an adversary w.r.t. $L$, i.e., an entity that plays against an implementation ensuring $S$ and that decides on the schedule and inputs of processes to win the game by having the implementation violate $L$. Our second necessary and sufficient condition states that there is a weakest liveness property that excludes safety property $S$ iff for all adversaries w.r.t. $L_{\max }$, the intersection of their behavior is also a behavior of an adversary w.r.t. $L_{\text {max }}$.

For all common safety properties studied in the literature and all common liveness properties, it is possible to find two adversaries the intersection of which behavior is not a behavior of an adversary, e.g. by considering two adversaries that make processes invoke operations with different arguments or different process identifiers, which results in two disjoint behaviors. Thus, for all common safety properties which are impossible to implement together with a corresponding strongest liveness property $L_{\max }$, we have two impossibility results. 
They basically state that there are no safety-liveness exclusion mappings.

A corollary of our impossibilities is, for instance, that there is no strongest liveness property that can be implemented (resp. no weakest liveness property which is impossible to implement) together with consensus safety, i.e., agreement and validity. Also, we can now answer questions such as the one raised in [4], about the existence of a weakest transactional memory (TM) liveness property which is impossible to implement together with opacity [15] and the existence of a strongest TM liveness property implementable with opacity*. We show that there are no such liveness properties. In fact, our impossibilities can be applied to many other contexts, such as k-set agreement [3] or high-level object implementations from registers [19] to show that there is no strongest implementable liveness property (resp. no weakest non-implementable liveness property).

One way to circumvent our impossibilities is to restrict the definitions of liveness and safety, i.e., basically to consider subsets $\mathscr{S} \subseteq \mathbb{S}$ and $\mathscr{L} \subseteq \mathbb{L}$, so that it is possible to find mappings $f_{w}: \mathbb{S} \rightarrow \mathscr{L}$ and $f_{s}: \mathbb{S} \rightarrow \mathscr{L}$. We explore a restricted definition of liveness that covers properties of non-blocking systems and includes properties that require either minimal or maximal progress and which are either dependent or independent of a scheduler. ${ }^{\dagger}$ Our restricted definition combines the notions of $k$-obstruction-freedom [35] and l-lockfreedom. While $k$-obstruction-freedom is a dependent maximal progress guarantee that requires progress of every process in a group of less than $k$ processes which execute alone, $l$-lock freedom is an independent minimal progress guarantee that requires progress of at least $l$ correct processes. We define a liveness property as a union of $l$-lock-freedom and $k$-obstruction-freedom, where $l \leq k$, and call such a property $(l, k)$-freedom. Being general enough, $(l, k)$-freedom covers liveness properties of shared objects considered most commonly used in literature and allows us to positively answer the safety-liveness exclusion question for many common safety properties. For example, considering consensus in a read-write shared memory system of $n$ processes, the strongest implementable liveness property is $(1,1)$-freedom and the weakest non-implementable is $(1,2)$ freedom; while for TM implementations the strongest implementable liveness property is $(1, n)$-freedom and the weakest non-implementable is $(2,2)$-freedom. Finally, we show that $(l, k)$-freedom has its own limitations by giving an example of a TM safety property for which we cannot find a weakest liveness that excludes the safety property.

The rest of the paper is organized as follows. Section 2 recalls basic definitions of a shared memory system. Section 3 defines safety and liveness properties. Section 4 poses the safety-liveness exclusion problem. There we give our impossibility results for the general case. Section 5 describes one way to circumvent the impossibility results. Section 6 concludes the paper by discussing alternative ways.

\section{System Model}

We consider a general shared memory system of $n$ asynchronous processes which might stop by crashing. The processes interact with each other only by performing atomic primitives on base objects. Base objects are shared objects, like read/write registers, test-and-set, compare-and-swap and etc., which are usually provided by the hardware and which are used to implement higher level shared objects. We assume that each process is sequential in the sense that after invoking a primitive, the process cannot invoke another primitive until it receives a corresponding response.

A shared object is defined by its type. A shared object type $T p$ is a tuple $(S t, \operatorname{Inv}, \operatorname{Res}, \operatorname{Seq})$, where $S t$ is the set of all possible states of the object, Inv is the set of all possible invocations on the object, Res is the set

\footnotetext{
*It was shown in [4] that in the case of TM shared objects [18, 21, 34], it is impossible to ensure both opacity and local progress, the strongest liveness property. However, the question whether there exists a weakest TM liveness property that excludes opacity or a strongest TM liveness property that does not exclude opacity remained open.

${ }^{\dagger}$ A non-blocking system is a one in which no process $p$ can prevent other processes from making progress once $p$ crashes, i.e. stops participating in a computation. A maximal [23] progress guarantee requires progress for all processes while a minimal [23] progress guarantee requires progress for some processes. A dependent [23] progress guarantee depends on the scheduling of processes while an independent [23] progress guarantee has the same requirement irrespectively of how processes are scheduled.
} 
of all possible responses from the object, and Seq $\subseteq$ Inv $\times S t \times S t \times$ Res is the sequential specification of the object. An implementation $I$ of a shared object of type $T p$ is a set of algorithms $\left\{I_{1}, \ldots, I_{n}\right\}$ on base objects such that each algorithm $I_{i}$ corresponds to a process $p_{i}$. When process $p_{i}$ invokes inv $v_{i}$ on $I$, where inv is some invocation from Inv, it executes algorithm $I_{i}$ with $i n v_{i}$ as an input of the algorithm. When executing algorithm $I_{i}$ process $p_{i}$ performs steps which could be an invocation, a response, or an atomic operation on base objects and local computations. The order in which processes take steps is determined by an external entity called a scheduler over which processes have no control. The system is asynchronous in the sense that a scheduler may delay any process for an arbitrary long period of time and there is no way for one process to determine if some other process is crashed or delayed.

We use the widely known I/O automata model of asynchronous shared memory systems [29] in which invocations and responses are input and output actions of an automaton. An I/O automaton $A$ is a 4-tuple $(\operatorname{states}(A), \operatorname{sig}(A), \operatorname{init}(A), \operatorname{trans}(A))[29,33]$, where:

- $\operatorname{states}(A)$ is a (finite or infinite) set of states,

- $\operatorname{init}(A) \subseteq \operatorname{states}(A)$ is a set of initial states,

- $\operatorname{sig}(A)=(\operatorname{in}(A), \operatorname{out}(A), \operatorname{int}(A))$ is an action signature, which partitions the set of all actions $\operatorname{acts}(A)=$ $\operatorname{in}(A) \cup \operatorname{out}(A) \cup \operatorname{int}(A)$ into input actions $\operatorname{in}(A)$, output actions $\operatorname{out}(A)$, and internal actions $\operatorname{int}(A)$,

- $\operatorname{trans}(A) \subseteq \operatorname{states}(A) \times \operatorname{acts}(A) \times \operatorname{states}(A)$ is a transition relation.

An execution of an $\mathrm{I} / \mathrm{O}$ automaton $A$ is a finite or infinite sequence of alternating states and actions $s_{0} \cdot a_{1}$. $s_{1} \cdot a_{2} \cdot s_{2} \cdot \ldots$ such that: (I) $s_{0} \in \operatorname{init}(A)$, (II) $\left(s_{i}, a_{i+1}, s_{i+1}\right) \in \operatorname{trans}(A)$ for every $i \in\{0,1,2, \ldots\}$, (III) if the sequence is finite, it should end with a state. The longest subsequence of an execution of $A$ consisting only of actions from $\operatorname{in}(A) \cup \operatorname{out}(A)$ is called a history. An action $a \in \operatorname{acts}(A)$ is enabled at a state $s \in$ states $(A)$ if there exists a state $s^{\prime} \in \operatorname{states}(A)$ such that $\left(s, a, s^{\prime}\right) \in \operatorname{trans}(A)$.

Let $A_{1}$ and $A_{2}$ be two I/O automata. Automata $A_{1}$ and $A_{2}$ are compatible if $\operatorname{out}\left(A_{1}\right) \cap \operatorname{out}\left(A_{2}\right)=\emptyset$, $\operatorname{int}\left(A_{1}\right) \cap \operatorname{acts}\left(A_{2}\right)=\emptyset$, and $\operatorname{int}\left(A_{2}\right) \cap \operatorname{acts}\left(A_{1}\right)=\emptyset$. The composition $A_{1} \times A_{2}$ of compatible I/O automata $A_{1}$ and $A_{2}$ is an $\mathrm{I} / \mathrm{O}$ automaton $A$ such that:

- $\operatorname{states}(A)=\operatorname{states}\left(A_{1}\right) \times \operatorname{states}\left(A_{2}\right)$,

- $\operatorname{init}(A)=\operatorname{init}\left(A_{1}\right) \times \operatorname{init}\left(A_{2}\right)$

- $\operatorname{sig}(A)=(\operatorname{in}(A), \operatorname{out}(A), \operatorname{int}(A))$ is such that:

$-\operatorname{int}(A)=\operatorname{int}\left(A_{1}\right) \cup \operatorname{int}\left(A_{2}\right) \cup\left(\operatorname{in}\left(A_{1}\right) \cap \operatorname{out}\left(A_{2}\right)\right) \cup\left(\operatorname{in}\left(A_{2}\right) \cap \operatorname{out}\left(A_{1}\right)\right)^{\ddagger}$,

- $\operatorname{in}(A)=\left(\operatorname{in}\left(A_{1}\right) \cup \operatorname{in}\left(A_{2}\right)\right) \backslash \operatorname{int}(A)$,

- out $(A)=\left(\operatorname{out}\left(A_{1}\right) \cup \operatorname{out}\left(A_{2}\right)\right) \backslash \operatorname{int}(A)$,

- $\left(\left(s_{1}, s_{2}\right), a,\left(s_{1}^{\prime}, s_{2}^{\prime}\right)\right) \in \operatorname{trans}(A)$ iff for every $i \in\{1,2\}$ the following holds:

- if $a \in \operatorname{acts}\left(A_{i}\right)$, then $\left(s_{i}, a, s_{i}^{\prime}\right) \in \operatorname{trans}\left(A_{i}\right)$,

- if $a \notin \operatorname{acts}\left(A_{i}\right)$, then $s_{i}=s_{i}^{\prime}$.

\footnotetext{
${ }^{\ddagger}$ Since in our model we assume invocations and responses with unique process identifiers, there is no invocation or response event which is associated with more than one process. Therefore, unlike [29, 33], we use a simplified definition of composition when input and output actions, that are used for communication between components, are hidden by becoming internal actions.
} 
An implementation $I=\left\{I_{1}, \ldots, I_{n}\right\}$ of a shared object of a type $T p=(S t, I n v$, Res, Seq $)$ is modeled by the composition $A_{I}=A_{I_{1}} \times \ldots \times A_{I_{n}} \times A_{B}$. Automaton $A_{B}$ models the behavior of all base objects used in the implementation (which can be represented as the composition of all automata corresponding to each base object). Each I/O automaton $A_{I_{i}}$ models the behavior of algorithm $I_{i}$ such that: states $\left(A_{I_{i}}\right)$ is the set of all states of $I_{i}$ and $\operatorname{init}\left(A_{I_{i}}\right)$ is the set of initial states of $I_{i},\left\{\operatorname{inv} v_{i} \mid \operatorname{inv} \in \operatorname{Inv}\right\} \subseteq \operatorname{in}\left(A_{I_{i}}\right),\left\{\right.$ res $_{i} \mid$ res $\left.\in \operatorname{Res}\right\} \subseteq$ $\operatorname{out}\left(A_{I_{i}}\right)$. Output actions from out $\left(A_{I_{i}}\right) \backslash\left\{i n v_{i} \mid i n v \in I n v\right\}$ are input actions of base objects and input actions from in $\left(A_{I_{i}}\right) \backslash\left\{\right.$ res $_{i} \mid$ res $\in$ Res $\}$ are output actions of base objects.

A process $p_{i}$ is pending in a history $h$ of $A_{I}$ if $h \mid p_{i}$ ends with an invocation, where $h \mid p_{i}$ is the longest subsequence of $h$ consisting only of acts $\left(A_{I_{i}}\right)$. Note that $h \mid p_{i}$ does not include input and output actions of $A_{I_{i}}$ which are used for communication with base objects modeled by $A_{B}$, this is so since such actions become internal in the composition $A_{I}=A_{I_{1}} \times \ldots \times A_{I_{n}} \times A_{B}$. Process $p_{i}$ is pending at a state $s$ if $s$ is reachable from an initial state by executing history $h$ in which $p_{i}$ is pending. An I/O automaton $A_{I}$ is input-enabled if for every process $p_{i}$, every invocation $i n v \in I n v$, and every state $s$ of $A_{I}$ the following holds: if process $p_{i}$ is not pending at state $s$, then $i n v_{i}$ is enabled at state $s$. Let $h_{i}$ be a history consisting only of actions from $\left\{i n v_{i} \mid i n v \in I n v\right\} \cup\left\{r e s_{i} \mid r e s \in R e s\right\}$, then history $h_{i}$ is well-formed, if $h$ is a sequence of alternating invocations and responses starting with an invocation. A history $h$ of an I/O automaton $A_{I}$ is well-formed if for every $A_{I_{i}}$ history $h \mid p_{i}$ is well-formed. Herein, we consider only well-formed histories and require each I/O automaton $A_{I_{i}}$ to be input-enabled.

To model process crashes, we augment the model with a special input action $\operatorname{crash}_{i}$ [29] and a special state $s_{\text {crashed }, i}$ of $A_{I_{i}}$ corresponding to each process $p_{i}$. At state $s_{\text {crashed, } i}$, no action of $A_{I_{i}}$ is enabled, and from every state $s \neq s_{\text {crashed }, i}$ of $A_{I_{i}}$, there is a transition $\left(s, \operatorname{crash}_{i}, s_{\text {crashed }, i}\right)$. When an I/O automaton $A_{I_{i}}$ executes action $\mathrm{Crash}_{i}$, it means that process $p_{i}$ crashes and stops executing any steps. Process $p_{i}$ crashes in history $h$, if $h$ has a $c r a s h_{i}$ action. Otherwise, process $p_{i}$ is said to be correct in history $h$. For every object type $T p=(S t, I n v, \operatorname{Res}, \operatorname{Seq})$ we denote by $\operatorname{ext}_{i}(T p)$ the set $\left(\left\{\operatorname{inv}_{i} \mid i n v \in \operatorname{Inv}\right\} \cup\left\{\operatorname{crash}_{i}\right\} \cup\left\{\operatorname{res}_{i} \mid \operatorname{res}_{\operatorname{ros}} \in \operatorname{Res}\right\}\right)$ and by $\operatorname{ext}(T p)$ the set $\bigcup_{1 \leq i \leq n} \operatorname{ext}_{i}(T p)$.

\section{Correctness properties}

\subsection{Safety}

A safety property states that some 'bad' events will never happen. For example, linearizability [24], serializability [31], and opacity [15], safety properties of shared memory systems, require that processes never receive responses which result in an inconsistent view of a shared memory system. A safety property is defined as a prefix-closed and limit-closed set of histories [1,29].

Definition 3.1. A safety property $S$ of a shared object of type $T p=(S t, I n v, R e s, S e q)$ is a non-empty set of histories consisting of actions from ext $(T p)$ such that (1) if $h \in S$, then every prefix of $h$ is in $S$, and (2) if $h_{1}, h_{2}, \ldots$ is an infinite sequence of finite histories in $S$ such that $h_{j}$ is a prefix of $h_{j+1}$, for every $j$, then the unique infinite history which is the limit of $h_{1}, h_{2}, \ldots$ is also in $S$. Implementation I of $T p$ ensures safety property $S$ if every finite history of $A_{I}$ is in $S$.

We assume that each safety property does not contain histories which cannot be implemented, i.e. we assume safety properties $S$ such that for any history $h \in S$, there exists an implementation $I$ such that $h$ is a history of $A_{I}$ and $I$ ensures $S$. We also make an additional assumption that a safety property should allow at least one response for any invocation that executes sequentially from an initial state. Specifically, we require that for each $i n v \in I n v$ and each process $p_{i}$ there exists res $\in$ Res such that $i n v_{i} \cdot r e s_{i} \in S$.

\subsection{Liveness}

A liveness property states that some 'good' events will eventually happen. For example, wait-freedom [19, 23] states that every operation by a correct process should eventually return a response. Starvation-freedom [2, 23] 
states that every correct process that tries to acquire a lock should eventually succeed. Local progress [4] states that every correct process should eventually commit its transactions.

A liveness property is a property which permits every finite history [30, 1, 29]. For I/O systems, a liveness property states that an input is eventually returned a corresponding output [33]. In particular, for shared memory systems, a liveness property states that some process is eventually returned a desirable response, i.e. makes progress $[23,25,35]$. For each shared object type, there is a property that requires progress for all correct processes, the strongest liveness requirement that can be expected. For example, for shared registers, the strongest liveness requirement is wait-freedom. Starvation-freedom is the strongest liveness requirement for lock-based implementations. For TM objects, the strongest liveness requirement is local progress. Hence, for every shared object type we assume a property $L_{\max }$ which states the strongest liveness requirement. Formally, with each shared object type $T p=(S t, I n v, \operatorname{Res}, S e q)$ we associate some set $L_{\max }$ of histories consisting of actions from $\operatorname{ext}(T p)$ such that every finite history $h$ consisting of actions from ext $(T p)$ is a prefix of some history in $L_{\max }$, i.e. there exists a history $h^{\prime}$ such that $h \cdot h^{\prime} \in L_{\max }$. Like fairness properties are defined in [38], we define each liveness property as a weakening of $L_{\max }$ :

Definition 3.2. A set $L$ of histories consisting of actions from ext $(T p)$ is a liveness property of a shared object of type $T p$ if $L_{\max } \subseteq L$.

Let $I$ be an implementation of an object of type $T p$. Intuitively, implementation $I$ ensures liveness property $L$ if every fair history of $A_{I}$ is in $L$. The restriction to fair histories is necessary because a liveness property cannot require progress from processes which do not get fair turns from a scheduler to perform steps [10]. An execution $e$ of $\mathrm{I} / \mathrm{O}$ automaton $A_{I}$ is fair if any of the following holds: (I) if $e$ is finite, then no action, other than crash actions, is enabled in the final state of $e$, or (II) if $e$ is infinite, then for every process $p_{i}$ execution $e$ has either infinitely many actions of $A_{I_{i}}$ or infinitely many occurrences of states at which no action, other than rrash $_{i}$, of $A_{I_{i}}$ is enabled. A history $h$ of $A_{I}$ is fair if there exists a fair execution $e$ of $A_{I}$ such that $h$ is the longest subsequence of $e$ consisting only of input and output actions. Denote by fair $\left(A_{I}\right)$ the set of all fair histories of $A_{I}$. Implementation I ensures liveness property $L$ if $\operatorname{fair}\left(A_{I}\right) \subseteq L$.

The stronger/weaker relation on properties is defined in the standard way [38]. Property $L_{1}$ is weaker than property $L_{2}\left(L_{2}\right.$ is stronger than $\left.L_{1}\right)$ if every implementation that ensures $L_{2}$ also ensures $L_{1}$. It easy to see that $L_{1}$ is weaker than $L_{2}$ iff $L_{2} \subseteq L_{1}$.

\section{Safety-liveness Exclusion}

A liveness property states that some good events should eventually happen, while a safety property states that some bad events should never happen. Safety and liveness properties exclude each other when the bad events of the safety property coincide with the good events of the liveness property. In the case of shared memory systems, this translates into the impossibility of implementing a shared object that ensures both properties. Let $S$ and $L$ be respectively a safety and a liveness properties of a shared object of type $T p$.

Definition 4.1. Liveness property $L$ excludes safety property $S$ if there is no implementation I of an object of type $T p$ such that I ensures both $S$ and $L$.

For example, if $T p$ is the consensus object type and the set of all possible implementations of $T p$ is restricted to implementations that use only read-write registers as base objects, then the impossibility result in $[8,5,28]$ states that wait-freedom excludes agreement and validity. Another example is the exclusion of local progress and opacity for transactional memory object type [4]. Below we address the following questions: when is it possible to determine a weakest (resp. a strongest) liveness property which excludes (resp. does not exclude) a given safety property? Basically we ask the following question: if $\mathbb{S}$ and $\mathbb{L}$ are the sets of all safety and liveness properties under given general definitions, what is the largest subset $\mathscr{S} \subseteq \mathbb{S}$ for which there exists a map $f_{w}: \mathscr{S} \rightarrow \mathbb{L}$ (resp. $f_{s}: \mathscr{S} \rightarrow \mathbb{L}$ ) that maps every safety property $S \in \mathscr{S}$ onto the weakest (resp. strongest) liveness property $L \in \mathbb{L}$ that excludes (resp. does not exclude) $S$. 


\subsection{Weakest non-implementable liveness}

Definition 4.2. A liveness property $L$ is the weakest liveness property to exclude a safety property $S$ if (1) $L$ excludes $S$ and (2) for any liveness property $L^{\prime}$, if $L^{\prime}$ excludes $S$ then $L^{\prime}$ is stronger than $L$.

In order to reason about the existence of a weakest liveness property that excludes $S$, we define a subset of $S$ which we call an adversary set. Informally, an adversary set w.r.t. $L$ and $S$ is a set of histories such that for every implementation $I$ ensuring $S$ there exists a history of $I$ which is in the adversary set and not in $L$. The existence of an adversary set w.r.t. $L$ and $S$ implies that there is an adversary, which decides on a sequence of steps produced by a scheduler and on invocations sent to implementation $I$, such that for any implementation the adversary makes $I$ produce an execution that violates $L$.

Definition 4.3. An adversary set w.r.t. $L$ and $S$ is a non-empty set of histories $F$ such that: (1) $F \subseteq S$, (2) $F \subseteq \bar{L}$, where $\bar{L}$ is the complement of $L$ taken over all well-formed histories, and (3) for every implementation I ensuring $S$ there is a history $h \in$ fair $\left(A_{I}\right)$ such that $h \in F$.

It is easy to see that $L$ excludes $S$ iff there is an adversary set w.r.t. $L$ and $S$. The following theorem gives a characterization of safety properties for which it is possible to find a weakest liveness property that excludes them.

Theorem 4.4. Let $F\left(L_{\max }\right)$ be the set of all adversary sets w.r.t. $L_{\max }$ and $S$, and let $G_{\max }=\bigcap_{F \in F\left(L_{\max }\right)} F$. There exists a weakest liveness property that excludes $S$ iff $G_{\max } \in F\left(L_{\max }\right)$.

Proof. Necessary condition: Let $L_{w}$ be the weakest liveness property that excludes $S$. Since $L_{w}$ excludes $S$, there exists an adversary set $F_{L_{w}}$ w.r.t. $L_{w}$ and $S$.

We first prove that $L_{w}=\overline{F_{L_{w}}}$. Assume that $L_{w} \neq \overline{F_{L_{w}}}$. By the definition of $F_{L_{w}}, F_{L_{w}} \subseteq \overline{L_{w}}$, and therefore $L_{w} \subseteq \overline{F_{L_{w}}}$. Because $L_{w} \neq \overline{F_{L_{w}}}$ and $L_{w} \subseteq \overline{F_{L_{w}}}$, there is a history $h$ such that $h \in \overline{F_{L_{w}}}$ and $h \notin L_{w}$. Because $h \in \overline{F_{L_{w}}}$ and $L_{w} \subseteq \overline{F_{L_{w}}}$, then $L_{w} \cup\{h\} \subseteq \overline{F_{L_{w}}}$, and consequently $F_{L_{w}} \subseteq \overline{L_{w} \cup\{h\}}$. Consider liveness property $L_{w} \cup\{h\}$,

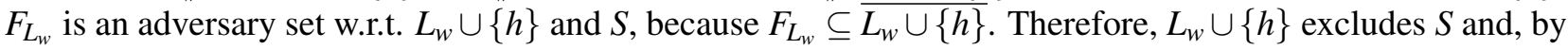
the definition of $L_{w}, L_{w} \cup\{h\}$ is stronger than $L_{w}$, i.e. $L_{w} \cup\{h\} \subseteq L_{w}$. This contradicts the fact that $h \notin L_{w}$.

Next, we prove that for every $F \in F\left(L_{\max }\right), F_{L_{w}} \subseteq F$. Assume that there is $F \in F\left(L_{\max }\right)$ such that $F_{L_{w}} \nsubseteq F$. Consider set $\bar{F}, \bar{F}$ is a liveness property because $F \subseteq \overline{L_{\max }}$ (by the definition of $F$ ), and therefore $L_{\max } \subseteq \bar{F}$. Since $F \subseteq \bar{F}$, then $F$ is an adversary set w.r.t. $\bar{F}$ and $S$, i.e., $\bar{F}$ excludes $S$. Since $\bar{F}$ excludes $S, \bar{F}$ is stronger than $L_{w}$, i.e. $\bar{F} \subseteq L_{w}$. Since $F_{L_{w}} \nsubseteq F$ and $L_{w}=\overline{F_{L_{w}}}$, then there exists a history $h^{\prime}$ such that $h^{\prime} \notin L_{w}$ and $h^{\prime} \in \bar{F}$. This contradicts the fact that $\bar{F} \subseteq L_{w}$.

Since $F_{L_{w}} \subseteq F$ for every $F \in F\left(L_{\max }\right)$, then $F_{L_{w}} \subseteq G_{\max }$. By the definition of $F_{L_{w}}$, for every implementation $I$ there is $h^{\prime \prime} \in \operatorname{fair}\left(A_{I}\right)$ such that $h^{\prime \prime} \in F_{L_{w}} \subseteq G_{\max }$. Thus, $G_{\max }$ is an adversary set w.r.t. $L_{\max }$ and $S$.

Sufficient condition: Because $G_{\max } \subseteq \overline{L_{\max }}$ (by the definition of an adversary set $G_{\max }$ ), then $L_{\max } \subseteq \overline{G_{\max }}$, i.e. $\overline{G_{\max }}$ is a liveness property. Since $G_{\max } \subseteq \overline{\overline{G_{\max }}}$ and $G_{\max } \in F\left(L_{\max }\right)$, then $G_{\max }$ is an adversary set w.r.t. $\overline{G_{\max }}$ and $S$. Hence, $\overline{G_{\max }}$ excludes $S$.

Consider some liveness property $L$ which excludes $S$. There is an adversary set $F_{L}$ w.r.t. $L$ and $S$. Since $L_{\max } \subseteq L$ and $F_{L} \subseteq \bar{L}$, then $F_{L} \subseteq \overline{L_{\max }}$. Therefore, $F_{L}$ is an adversary set w.r.t. $L_{\max }$ and $S$. Since $G_{\max }$ is the intersection of all adversary sets w.r.t. $L_{\max }$ and $S, G_{\max }$ is a subset of every adversary set w.r.t. $L_{\max }$ and $S$. From $G_{\max } \subseteq F_{L} \subseteq \bar{L}$ it follows that $L \subseteq \overline{G_{\max }}$. Thus, we showed that every liveness property that excludes $S$ is stronger than $\overline{G_{\max }}$. By definition, $\overline{G_{\max }}$ is the weakest liveness property that excludes $S$.

For every object type $T p$ and most symmetric safety properties $S$ of $T p$, i.e. safety properties the requirements of which are the same for all processes irrespectively of process identifiers, it is possible to find two adversary sets $F_{1}$ and $F_{2}$ w.r.t. $L_{\max }$ and $S$ such that $F_{1} \cap F_{2}=\emptyset$, and consequently $G_{\max } \notin F\left(L_{\max }\right)$. Therefore, Theorem 4.4 means that for most safety properties there is no weakest liveness property that excludes the given safety property. We have the following two corollaries: 
Corollary: consensus implementations from registers. A consensus shared object is used by processes to agree on some value from a set of proposed values. Each process proposes its own value $v$ by invoking operation propose $(v)$ on a consensus object and receives as a response some value $v^{\prime}$ (decides value $v^{\prime}$ ). Formally, agreement and validity, a safety property of consensus objects, states that all processes decide the same value and the decided value is the value proposed by one of the processes. Waitfreedom [19], a liveness property of consensus objects, states that every correct process eventually decides. Consider implementations of consensus that use only read/write registers as base objects. In that case, it is shown in [5] that if two processes propose different values, then there is an execution in which both of them take infinite number of steps and at least one of them does not decide a value. Hence, there exists an adversary set $F_{1}=\left\{\right.$ propose $_{1}(v) \cdot$ propose $_{2}\left(v^{\prime}\right)$, propose $_{1}(v) \cdot v_{1} \cdot$ propose $_{2}\left(v^{\prime}\right)$, propose $_{1}(v) \cdot$ propose $_{2}\left(v^{\prime}\right)$. $\left.v_{1}, \operatorname{propose}_{1}(v) \cdot \operatorname{propose}_{2}\left(v^{\prime}\right) \cdot v_{1}^{\prime}, \operatorname{propose}_{1}(v) \cdot \operatorname{propose}_{2}\left(v^{\prime}\right) \cdot v_{2}, \operatorname{propose}_{1}(v) \cdot \operatorname{propose}_{2}\left(v^{\prime}\right) \cdot v_{2}^{\prime}\right\}$ w.r.t. waitfreedom and agreement and validity, i.e. $F_{1}$ is the set of all histories in which two processes propose different value and one of those two processes does not decide. The proof of the impossibility result does not depend on whether process $p_{1}$ invokes the propose operation first or not, consequently, the set of histories, in which $p_{2}$ invokes propose before $p_{1}, F_{2}=\left\{\right.$ propose $_{2}(v) \cdot$ propose $_{1}\left(v^{\prime}\right)$, propose $_{2}(v) \cdot v_{2} \cdot$ propose $_{1}\left(v^{\prime}\right)$, propose $_{2}(v)$. propose $_{1}\left(v^{\prime}\right) \cdot v_{2}, \operatorname{propose}_{2}(v) \cdot \operatorname{propose}_{1}\left(v^{\prime}\right) \cdot v_{2}^{\prime}, \operatorname{propose}_{2}(v) \cdot \operatorname{propose}_{1}\left(v^{\prime}\right) \cdot v_{1}, \operatorname{propose}_{2}(v) \cdot \operatorname{propose}_{1}\left(v^{\prime}\right) \cdot$ $\left.v_{1}^{\prime}\right\}$ is also an adversary set. Since $F_{1} \cap F_{2}=\emptyset$, it follows that $G_{\max }=\emptyset$.

Corollary 4.5. There is no weakest liveness property of consensus objects which excludes agreement and validity when these objects are implemented only from read/write registers.

Corollary: transactional memory. Transactional memory (TM) allows to enclose sequential code within atomic transactions which are executed concurrently. Transactional code contains accesses to transactional variables which can be read or written, these variables can be accessed only within transactions. Each transaction is executed only by one process. Processes in a TM implementation can invoke the following transactional operations: start which requests to start a new transaction and returns either $o k$ or an abort event $A, x$.write $(v)$ which writes value $v$ to transactional variable $x$ within a transaction and returns either $o k$ or an abort event $A$; $x$.read which reads a value from transactional variable $x$ within a transaction and returns either a value $v$ or an abort event $A$; try $C$ which requests to commit a transaction and returns a commit event $C$ or an abort event $A$. The sequential specification $S e q$ of a TM object is such that if a transaction commits, then all the changes made to transactional variables within the transaction are made visible to other processes, and if a transaction aborts, then all the changes are discarded.

Opacity [15], a safety property of TM implementations, states that every transaction, even aborted, observes a consistent state of the system. Specifically, history $h$ ensures opacity if for every finite prefix $h^{\prime}$ of $h$ there exists a sequential history $s$ such that $s$ is equivalent to some completion $\operatorname{comp}\left(h^{\prime}\right)$ of $h^{\prime}, s$ preserves the real time order of $\operatorname{comp}\left(h^{\prime}\right)$, and $s$ respects the sequential specification Seq. A completion comp $(h)$ of history $h$ is any history derived from $h$ by appending $\operatorname{try} C \cdot A$ for every transaction which does not invoke a commit request in $h$ and by appending either an abort event $A$ or a commit event $C$ for every transaction which invokes a commit request but does not receive a corresponding response in $h$. Two histories $h_{1}$ and $h_{2}$ are equivalent if for every process $p_{i}, h_{1}\left|p_{i}=h_{2}\right| p_{i}$. History $h_{1}$ preserves the real time order of history $h_{2}$ if for any two transaction $T_{1}$ and $T_{2}$ in $h_{2}$ if $T_{1}$ receives an abort or a commit event, i.e. completes, before $T_{2}$ invokes start in $h_{2}$, then $T_{1}$ completes before $T_{2}$ starts in $h_{1}$. In TM implementations requiring that each operation returns a response is not enough because such requirement can be trivially ensured simply by aborting every transaction. To make progress transactions should be able to eventually commit. Therefore, the set of 'good' events is restricted to commit events. Local progress [4], the strongest liveness property of TM implementations, requires that every correct process is eventually given a chance to invoke a commit request $\operatorname{try} C()$ and eventually one of the commit requests $\operatorname{try} C()$ is returned a commit response $C$. In other words local progress requires that for every correct process eventually there is a transaction which is not aborted. It was shown in [4] that it is impossible to implement a TM object which ensures both opacity and local progress. Consider the adversary set defined by the following strategy [4]: 
1. Step 1. Process $p_{1}$ invokes start 1 and waits until it receives as a response $o k_{1}$ or $A_{1}$. If the response is $A_{1}$ the adversary repeats Step 1. Otherwise, process $p_{1}$ invokes $x$.read ${ }_{1}()$ and waits until it receives as a response $v_{1}^{\prime}$ or $A_{1}$. If the response is $A_{1}$ the adversary repeats Step 1. Otherwise, the adversary goes to Step 2.

2. Step 2. Process $p_{2}$ invokes start 2 and waits until it receives as a response $o k_{2}$ or $A_{2}$. If the response is $A_{2}$ the adversary repeats Step 2. Otherwise, process $p_{2}$ invokes $x \cdot \operatorname{read}_{2}()$ and waits until it receives as a response $v_{2}^{\prime \prime}$ or $A_{2}$. If the response is $A_{2}$, the adversary repeats Step 2. Otherwise $p_{2}$ invokes $x$.write $e_{2}\left(v^{\prime}+1\right)$, and waits until it receives as a response $o k_{2}$ or $A_{2}$. If the response is $A_{2}$, then the adversary repeats Step 2 . Otherwise, $p_{2}$ invokes $\operatorname{try} C_{2}()$ operation and waits until it receives a response $C_{2}$ or $A_{2}$. If the response is $A_{2}$, the adversary repeats Step 2. Otherwise the strategy goes to Step 3.

3. Step 3. Process $p_{1}$ invokes $x$.write $e_{1}\left(v^{\prime \prime}+1\right)$ and waits until it receives as a response $o k_{1}$ or $A_{1}$. If the response is $A_{1}$, then the adversary goes to Step 1 . Otherwise $p_{1}$ invokes $\operatorname{try} C_{1}()$ operation and waits until it receives a response $C_{1}$ or $A_{1}$. If the response is $A_{1}$, the adversary goes to Step 1 . Otherwise the adversary stops.

Let $F_{1}$ be the set of all histories produced by the adversary set described by the above strategy, i.e. the set of histories that result from applying the strategy to every possible TM object implementation that ensures opacity. In [4] it is shown that every history which ensures opacity and which is produced by the above strategy violates local progress. Therefore, $F_{1}$ is an adversary set w.r.t. local progress and opacity. Let us exchange processes in the above strategy so that process $p_{1}$ plays the role of $p_{2}$ and vice versa and let $F_{2}$ be the set of all possible histories produced by the resulting adversary. Set $F_{2}$ is an adversary set w.r.t. local progress and opacity since the impossibility result does not depend on process identifiers. Since local progress is the strongest liveness property of TM objects, then $F_{1}, F_{2} \in F\left(L_{\max }\right)$. However $F_{1} \cap F_{2}=\emptyset$, because every history from $F_{1}$ begins with start ${ }_{1}$ invocation and every history from $F_{2}$ begins with start $_{2}$. Since $F_{1} \cap F_{2}=\emptyset$, it follows that $G_{\max }=\emptyset$.

Corollary 4.6. There is no weakest liveness property of TM objects which excludes opacity.

\subsection{Strongest implementable liveness}

Definition 4.7. Liveness property $L$ is the strongest liveness property that does not exclude a given safety property $S$ if (1) $L$ does not exclude $S$, and (2) for any liveness property $L^{\prime}$ if $L^{\prime}$ does not exclude $S$ then $L^{\prime}$ is weaker than $L$.

Before proving the theorem which states that $L_{\max }$ is the only possible strongest liveness property that does not exclude $S$ in case when such a property exists, we prove the following lemma first.

Lemma 4.8. The strongest liveness property that an implementation I ensures is $L_{\text {max }} \cup$ fair $\left(A_{I}\right)$.

Proof. By contradiction, assume that there is a liveness property $L$ such that $I$ ensures $L$ and $L_{\max } \cup$ fair $\left(A_{I}\right)$ is not stronger than $L$, i.e., $L_{\max } \cup \operatorname{fair}\left(A_{I}\right) \nsubseteq L L$. Because $L$ is a liveness property, $L_{\max } \subseteq L$. And because $I$ ensures $L$, then $\operatorname{fair}\left(A_{I}\right) \subseteq L$. From $L_{\text {max }} \subseteq L$ and fair $\left(A_{I}\right) \subseteq L$ it follows that $L_{\text {max }} \cup$ fair $\left(A_{I}\right) \subseteq L$. This contradicts the fact that $L_{\max } \cup \operatorname{fair}\left(A_{I}\right) \nsubseteq L$.

Theorem 4.9. If there is a strongest liveness property that does not exclude $S$, then it should be $L_{\max }$.

Proof. Let $L_{s}$ be the strongest liveness property that does not exclude $S$. By definition, there is an implementation $I_{S}$ which ensures both $L_{S}$ and $S$. By Lemma 4.8, $L_{S}=L_{\max } \cup \operatorname{fair}\left(A_{I_{s}}\right)$.

Assume that $L_{s} \neq L_{\max }$, i.e. fair $\left(A_{I_{s}}\right) \backslash L_{\max } \neq \emptyset$. We first prove that every history in fair $\left(A_{I_{s}}\right) \backslash L_{\max }$ does not include responses. Assume, by contradiction, that there is history $h \in \operatorname{fair}\left(A_{I_{s}}\right) \backslash L_{\max }$ which includes 
a response. Let $\operatorname{res}_{k}$ be the first response in $h$, i.e. $h=h^{\prime} \cdot \operatorname{res}_{k} \cdot h^{\prime \prime}$ and $h^{\prime}$ does not include any responses. Consider a trivial implementation $I_{t}$ which does not return responses for any invocation. Let $A_{I_{t}}$ be an I/O automaton that models $I_{t}$. Notice that since the implementation is trivial it does not require any base objects. Because $I_{t}$ does not return responses, then every history of $A_{I_{t}}$ consists only of invocations and probably some crash events, and therefore, history $h$ is not a history of $\operatorname{fair}\left(A_{I_{t}}\right)$. By Lemma 4.8 , implementation $I_{t}$ ensures liveness property $L_{t}=L_{\max } \cup$ fair $\left(A_{I_{t}}\right)$, note that $L_{t}$ is not weaker than $L_{s}$ since $h \notin \operatorname{fair}\left(A_{I_{t}}\right)$ and $h \in \operatorname{fair}\left(A_{I_{s}}\right) \backslash L_{\text {max }}$. Let $h_{t}$ be some history of $A_{I_{t}}$. Because $h_{t}$ consists only of invocations and probably some crash events, which are input actions of $A_{I_{s}}$, and $A_{I_{s}}$ is input-enabled, $h_{t}$ is also a history of $A_{I_{s}}$. Because $I_{s}$ ensures $S, h_{t} \in S$. Thus, every history of $A_{I_{t}}$ is in $S$ and therefore $I_{t}$ ensures $S$. Hence, $L_{t}$ does not exclude $S$. This contradicts the facts that $L_{s}$ is the strongest liveness property that does not exclude $S$ and $L_{t}$ is not weaker than $L_{s}$.

Next we prove that for any history $h \in \operatorname{fair}\left(A_{I_{s}}\right) \backslash L_{\max }$, for any process $p_{l}$, and for any response res $s_{l}$, $h \cdot$ res $_{l} \notin S$ holds. Assume, by contradiction, that there is a history $h \in \operatorname{fair}\left(A_{I_{s}}\right) \backslash L_{\max }$, a process $p_{l}$, a response $r e s_{l}$ such that $h \cdot r e s_{l} \in S$. Let $i n v_{l}$ be the invocation in $h$ corresponding to response resl, i.e. $h \cdot r e s_{l} \mid p_{l}=$ $i n v_{l} \cdot r e s_{l}$. Consider history inv $v_{l} \cdot r e s_{l} \in S$. Let $I$ be an implementation such that $I$ ensures $S$ and $i n v_{l} \cdot r e s_{l}$ is a history of $A_{I}$.

Consider a trivial implementation $I_{b}$ such that: (1) when algorithm $I_{l}^{b}$, corresponding to $p_{l}$, receives invocation $i n v_{l}$ the first time it returns $r e s_{l}$, and for the second instance of $i n v_{l}$ it stops without returning any response, (2) when $I_{l}^{b}$ receives an invocation other than $i n v_{l}$ it stops without returning any response, and (3) every algorithm $I_{j}^{b}$, where $j \neq l$, once it receives any invocation, it stops without returning any response. Formally, implementation $I_{b}$ can be described by an I/O automaton $A_{I_{b}}=A_{I_{1}^{b}} \times \ldots \times A_{I_{n}^{b}}$ (because $I$ does not use any base objects $A_{B}$ is omitted from the composition) such that:

- for every $1 \leq i \leq n, \operatorname{in}\left(A_{I_{i}^{b}}\right)=\left\{i n v_{i} \mid i n v \in \operatorname{Inv}\right\} \cup\left\{\operatorname{crash}_{i}\right\}$ and $\operatorname{out}\left(A_{I_{i}^{b}}\right)=\left\{\operatorname{res}_{i} \mid \operatorname{res} \in \operatorname{Res}\right\}$;

- when $A_{I_{l}^{b}}$ executes $i n v_{l}$ from an initial state, $A_{I_{l}^{b}}$ changes its state to a state $s^{l}$ at which only $\operatorname{crash}_{l}$ and output action res $s_{l}$ are enabled. Let $s_{e n}^{l}$ be a state of $A_{I_{l}^{b}}$ such that there is a transition $\left(s^{l}\right.$, res $\left.s_{l}, s_{e n}^{l}\right)$ in $A_{I_{l}^{b}}$; note that from state $s_{e n}^{l}$ every invocation is enabled. For every invocation $i n v^{\prime} \in I n v$, when $A_{I_{l}^{b}}$ executes $i n v_{l}^{\prime}$ from $s_{e n}^{l}, A_{I_{l}^{b}}$ changes its state to a state $s_{1}^{l}$ at which only $\operatorname{crash}_{l}$ action is enabled;

- for every invocation $i n v^{\prime \prime} \in I n v$, such that $i n v^{\prime \prime} \neq i n v$, when $A_{I_{l}^{b}}$ executes $i n v_{l}^{\prime \prime}$ from an initial state $A_{I_{l}^{b}}$ changes its state to a state $s_{2}^{l}$ at which only $\operatorname{crash}_{l}$ action is enabled;

- for every $j \neq l$ and for every invocation $i n v^{\prime} \in I n v$, when $A_{I_{j}^{b}}$ executes $i n v_{j}^{\prime}$ from an initial state, $A_{I_{j}^{b}}$ changes its state to a state $s_{1}^{j}$ at which only $\operatorname{crash}_{j}$ action is enabled.

Let $h^{\prime}$ be any history of $A_{I_{b}}$ such that $h^{\prime}$ contains a response. Because $I_{b}$ returns a response only for the first instance of $i n v_{l}$, it follows that the response in $h^{\prime}$ is res $s_{l}$ and inv $v_{l} \cdot r e s_{l}$ is a prefix of $h^{\prime} \mid p_{l}$. Because inv $v_{l} \cdot r e s_{l}$ is a history of $A_{I}$ and $A_{I}$ is input-enabled, then $h^{\prime}$ is also a history of $A_{I}$. Because $I$ ensures $S$, it follows that every such history $h^{\prime}$ is in $S$. Let $h^{\prime \prime}$ be any history of $A_{I_{b}}$ such that $h^{\prime \prime}$ does not contain a response. Because $A_{I}$ is input-enabled, $h^{\prime \prime}$ is also a history of $A_{I}$ and, consequently, $h^{\prime \prime} \in S$. Hence, every history of $A_{I_{b}}$ is in $S$ and, consequently, $I_{b}$ ensures $S$. Because (1) $h \mid p_{l}=i n v_{l}$, and (2) state $s^{l}$ of I/O automaton $A_{I_{l}^{b}}$ is reachable by executing $i n v_{l}$ from an initial state, and (3) at $s^{l}$ output action res $_{l}$ is enabled then every execution of $A_{I_{b}}$, to which history $h$ corresponds, is not fair, and consequently $h \notin \operatorname{fair}\left(A_{I_{b}}\right)$.

By Lemma 4.8, implementation $I_{b}$ ensures liveness property $L_{b}=L_{\max } \cup$ fair $\left(A_{I_{b}}\right)$. Hence, $L_{b}$ does not exclude $S$. Because $h \in L_{s}$ and $h \notin L_{b}$, liveness property $L_{b}$ is not weaker than liveness property $L_{s}$. This contradicts the facts that $L_{S}$ is the strongest liveness property that does not exclude $S$ and $L_{b}$ does not exclude $S$. 
We proved that for every history $h \in \operatorname{fair}\left(A_{I_{s}}\right) \backslash L_{\max }$ the following holds: (1) $h$ does not contain any responses and (2) there is no process $p_{i}$ and response res such that $h \cdot$ res $_{i} \in S$.

Let $h$ be any history in fair $\left(A_{I_{s}}\right) \backslash L_{\max }$ and let $i n v_{i}$ be some invocation in $h$ by a process $p_{i}$ which does not crash in $h$. Let res $s_{i}$ be a response such that inv $_{i} \cdot$ res $_{i} \in S$ and let $I^{\prime}$ be an implementation which ensures $S$ and which has $i n v_{i} \cdot$ res $_{i}$ as its history. Because $A_{I^{\prime}}$, an I/O automata model of $I^{\prime}$, is input-enabled, it follows that every history $h^{\prime}$ such that $h^{\prime}$ includes a single response and $h^{\prime} \mid p_{i}=i n v_{i} \cdot$ res $_{i}$ is a history of $A_{I^{\prime}}$. Because $I^{\prime}$ ensures $S$, it follows that every such history $h^{\prime} \in S$. This contradicts the fact that $h \cdot r^{r e s} s_{i} \notin S$.

Corollary: consensus objects. Consider those implementations of consensus that use only read/write registers as base objects. Since $L_{\max }$, which is wait-freedom, is impossible [5] to ensure together with agreement and validity using only registers, then we have the following corollary:

Corollary 4.10. There is no strongest liveness property of consensus objects which does not exclude agreement and validity when these objects are implemented only from read/write registers.

Corollary: transactional memory. Because $L_{\max }$, which is local progress, is impossible [4] to ensure together with opacity, then we have the following corollary:

Corollary 4.11. There is no strongest liveness property of TM objects which does not exclude opacity.

\section{Circumventing the Impossibilities}

In this section we show that if we restrict the space of liveness properties, then it is possible to determine weakest (strongest) liveness property that excludes (does not exclude) a safety property in certain cases.

\section{$5.1(l, k)$-Freedom}

Instead of giving a general set-theoretic definition of liveness, we give a definition based on the notion of progress [23]. Accordingly, liveness properties can be classified into properties which require either maximal or minimal progress and which are either dependent or independent of a scheduler. Maximal progress properties require progress for every correct process, while minimal progress properties require progress for some correct processes. Wait-freedom, which requires progress for every correct process, and obstructionfreedom, which requires progress only for processes that eventually run without step contention, are examples of maximal progress properties. Lock-freedom, which requires progress for at least one correct process, is an example of a minimal progress property. Wait-freedom and lock-freedom are examples of independent progress properties, while obstruction-freedom is an example of dependent progress property. We give a definition of liveness that encompasses both maximal and minimal progress and dependent and independent guarantees.

Intuitively, we say that a process makes progress in an execution if it eventually receives 'good' responses in that execution for its invocations. However, for different object types, the notion of a 'good' response might be different. For example, for objects like consensus or registers, any response is a 'good' response; but for TM objects 'good' responses are those which do not abort transactions. Therefore, we assume that for each object type $T p=(S t, I n v, \operatorname{Res}, S e q)$ there is a fixed subset of responses $G_{T p} \subseteq$ Res which are necessary to make progress. We say that a correct process $p_{i}$ makes progress in a fair execution $e$ if $e$ contains infinitely many responses from $\left\{\right.$ res $_{i} \mid$ res $\left.\in G_{T p}\right\}$.

Between the independent progress guarantee of wait-freedom and the dependent progress guarantee of obstruction-freedom lie intermediate maximal progress guarantees that can be satisfied only when up to $k$ processes are scheduled to take steps. Such progress guarantees are grouped into $k$-obstruction-freedom properties. $k$-Obstruction-freedom [35] states that if at any point in an execution there are at most $k$ processes taking steps, then all these processes should make progress. Likewise, between the maximal progress guarantee of wait-freedom and the minimal progress guarantee of lock-freedom lie intermediate independent 
progress guarantees that require progress for at least $l$ processes. We introduce the notion of $l$-lock-freedom to group such guarantees. $l$-Lock-freedom states that at least $l$ processes should make progress if there are at least $l$ correct processes in an execution or all processes should make progress if there are less than $l$ correct processes in an execution.

$l$-Lock-freedom and $k$-obstruction-freedom are different kinds of progress requirements. We combine these two kinds of requirements into a more general one. Specifically, we define $(l, k)$-freedom, where $l \leq k$.

Definition 5.1. A fair execution e ensures $(l, k)$-freedom if the following holds. If at most $k$ processes take infinitely many steps in e, then

- if at least l processes are correct in e, then at least l processes make progress in e,

- if less than $l$ processes are correct in e, then all correct processes make progress in $e$.

Observe that if a process is correct in $e$ (i.e. it does not crash) it does not necessary mean that it takes infinitely many steps in $e$, e.g. a correct process might be prevented from taking steps by the implementation which does not enable steps after certain state. Notice that if $L F_{l}$ is the set of all executions that ensure $l$-lockfreedom and $O F_{k}$ is the set of all executions that ensure $k$-obstruction-freedom, then $L F_{l} \cup O F_{k}$ is the set of all executions that ensure $(l, k)$-freedom. An implementation $I$ is $(l, k)$-free if every fair execution of $I$ ensures $(l, k)$-freedom.

Liveness properties defined as $(l, k)$-freedom are not totally ordered. For example, consider $(1,3)$-freedom and $(2,2)$-freedom properties. An execution in which only two processes take steps and only one of those two processes makes progress ensures (1,3)-freedom but does not ensure (2,2)-freedom. An execution in which only three processes take steps and none of those three processes makes progress ensures $(2,2)$-freedom but does not ensure (1,3)-freedom. Therefore, (1,3)-freedom and (2,2)-freedom are incomparable to each other. However, despite the fact that $(l, k)$-freedom properties are not totally ordered, it is possible to find weakest non-implementable and strongest implementable $(l, k)$-freedom properties in most cases.

\subsection{Consensus and TM examples}

Consider implementations of consensus that use only read/write registers as base objects. The impossibility of consensus from registers [5] says that if two processes invoke two propose $(v)$ operations with different arguments, then there is an adversary set that produces a fair execution in which none of the two processes ever decides a value. Consequently, (1,2)-freedom excludes agreement and validity in the case of implementations from registers. Because $(1,1)$-freedom, which is obstruction-freedom, is the only consensus liveness property which is weaker than $(1,2)$-freedom and which is possible $[20,17]$ to implement using registers, we have the following theorem.

Theorem 5.2. For implementations of consensus from read/write registers $(1,1)$-freedom is the strongest $(l, k)$-freedom property that does not exclude agreement and validity and $(1,2)$-freedom is the weakest $(l, k)$ freedom property that excludes agreement and validity.

As another example, consider TM shared objects. In [4] it is shown that any non-blocking liveness property which is biprogressing is impossible to implement in the TM context with safety properties like strict serializability [31] or opacity [15] in a system of two processes. A biprogressing liveness property is a property which requires progress for at least two correct processes. In the case of $(l, k)$-freedom properties, the weakest biprogressing property is $(2,2)$-freedom. Since in the TM context it is possible [9] to implement $(1, n)$-freedom, which is the strongest property that requires progress for at most one process, together with opacity, we have the following theorem.

Theorem 5.3. For TM implementations $(1, n)$-freedom is the strongest $(l, k)$-freedom property that does not exclude opacity and $(2,2)$-freedom is the weakest $(l, k)$-freedom property that excludes opacity. 
It is worth noting that in case of $(l, k)$-freedom liveness properties, the strongest implementable TM liveness property, $(1, n)$-freedom, is not weaker than the weakest non-implementable TM liveness property, $(2,2)$ freedom. In fact, these two properties are incomparable.

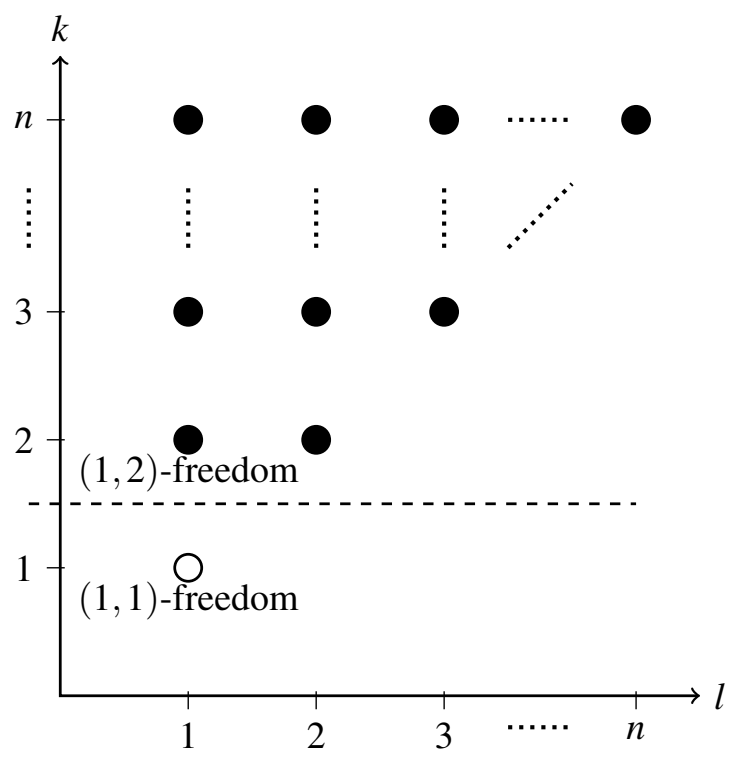

(a) $S$ is agreement and validity of consensus.

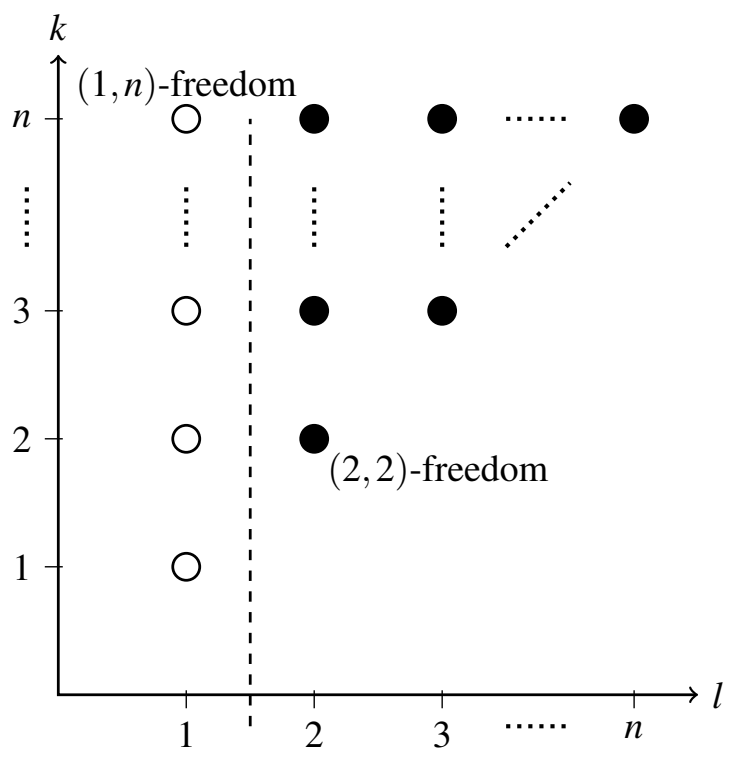

(b) $S$ is opacity of TM.

Figure 1: $(l, k)$-Freedom properties can be represented as points on a two-dimensional plane. The more a point located to the right and the higher it is, the stronger the corresponding $(l, k)$-freedom property is. White points indicate $(l, k)$-freedom properties that do not exclude a given safety property $S$, and black ones exclude $S$.

\subsection{Limitations of $(l, k)$-freedom: a counterexample}

Even though $(l, k)$-freedom allows to circumvent the safety-liveness exclusion impossibility for consensus and TM from Section 4, there are still some safety properties for which it is not the case. For instance, consider the following safety property $S$ of TM. A TM history $h$ ensures $S$ if the following hold:

1. $h$ ensures opacity, and

2. for any three (or more) concurrent transactions $T_{1}, T_{2}, T_{3}, \ldots$ in $h$, executed by different processes $p_{1}, p_{2}, p_{3}, \ldots$, respectively, if (1) there exists a number $t$ s.t. for each process $p_{i} \in\left\{p_{1}, p_{2}, p_{3}, \ldots\right\}$, $T_{i}$ is the $t$-th transactions in $h \mid p_{i}$ and (2) each $T_{i}$ invokes $\operatorname{try} C()_{i}$ after at least other two transactions from $\left\{T_{1}, T_{2}, T_{3}, \ldots\right\}$ receive a response for a $\operatorname{start}()_{j}$ operation in $h$, where $j \neq i$, then $T_{1}, T_{2}, T_{3}, \ldots$ should be aborted in $h$.

In other words, safety property $S$ requires opacity plus an additional requirement that if three or more transactions have the same timestamp $t$ and invoke a commit request $\operatorname{try} C()$ after any two of them receive a response for starting a transaction request start(), then such transactions should be aborted. In [4] it is shown that $(2,2)$-freedom is impossible to implement together with opacity. Because $S$ includes the requirement of opacity, then in the TM context $(2,2)$-freedom excludes $S$. In order to show that $(1,3)$-freedom excludes $S$, consider the following adversary:

1. Step 1. Processes $p_{1}, p_{2}, p_{3}$ concurrently invoke $\operatorname{start}()$ request to start a transaction and each waits for a response which should be either $o k$ or $A$. Once each of the three processes receives a response, the adversary goes to Step 2 . 
2. Step 2. Processes which did not receive an abort event $A$ at Step 1 , concurrently invoke $\operatorname{try} C()$ request to commit their transaction and each waits for a response which should be either $C$ or $A$. If each process receives an abort event $A$, the adversary goes to Step 1 . Otherwise the adversary stops.

Assume that the adversary terminates, i.e. there exists a history $h$ such that some process commits in $h$. Without loss of generality assume this process to be $p_{1}$. Let the committing transaction $T_{1}$ be a $t$-th transaction of $p_{1}$. According to the adversary, the three processes invoke new transactions concurrently to each other, and consequently the committing transaction is concurrent to the other two $t$-th transactions of $p_{2}$ and $p_{3}$. According to the adversary, $T_{1}$ invokes $\operatorname{try} C()$ only after $p_{2}$ and $p_{3}$ receive responses for start () at Step 1 (which eventually happens because of $(1,3)$-freedom). Thus the conditions of the second requirement of property $S$ are satisfied, and consequently, transactions $T_{1}, T_{2}, T_{3}$ should be aborted - a contradiction. Since the adversary never terminates, none of the three correct processes receives a commit response. Therefore the history produced by the adversary violates $(1,3)$-freedom.

In order to show that there is no weakest $(l, k)$-freedom property non-implementable with $S$ we show that $(1,2)$-freedom, which is weaker than both $(1,3)$-freedom and $(2,2)$-freedom, is implementable with $S$. Below we give a simple TM implementation, which is a modification of algorithm $A_{G P}$ from [16], the purpose of which is to show that $(1,2)$-freedom does not exclude $S$.

The main idea of the implementation is the following. The implementation uses a single shared compareand-swap object which holds a version number and a value of each transactional variable. Additionally, it uses a shared snapshot object of $n$ registers, where $n$ is the total number of processes. When a process $p_{i}$ starts a new transaction, it increments its local timestamp, writes the new timestamp to the $i$-th register, and copies the content of the compare-and-swap object to its local memory. The process performs transactional reads and writes using only the local memory. When the process invokes a commit request it first takes the snapshot of the registers and checks if at least two other processes have a greater timestamp. If so, the transaction is aborted. Otherwise, the process tries to update the compare-and-swap object with a new version number and new values from its local memory. The version numbers are used to ensure opacity, while the timestamps are used to ensure the second requirement of $S$.

Lemma 5.4. Algorithm $I_{(1,2)}$ implements a TM that ensures $S$ and (1,2)-freedom.

Proof. Consider any history $h$ of $I_{(1,2)}$. Within this proof, we say that a transaction $T_{k}$ reads version $w$ if $T_{k}$ is returned in start $_{i}$ a tuple (version,oldval), where version $=w$. We say that $T_{k}$ commits version $w$ if $T_{k}$ reads version $w-1$ and is returned value true from operation compare-and-swap on $C$ in $\operatorname{try} C()$.

The first requirement of $S$ (Opacity). Observe that the version number stored in object $C$ can only increase with time. Hence, if a transaction $T_{i}$ precedes a transaction $T_{k}$ in $h$, then $T_{i}$ cannot read a version higher than the version read by $T_{k}$. Note also that from any set of transactions that read the same version $w$ at most one transaction can commit version $w+1$. Therefore, there exists a total order $\ll$ on the set of transactions in $h$, such that, for all transactions $T_{i}$ and $T_{k}$ in $H, T_{i} \ll T_{k}$ if:

- $T_{i}$ precedes $T_{k}$ in $h$; or

- $T_{i}$ reads a version $w_{i}, T_{k}$ reads a version $w_{k}$, and $w_{i}<w_{k}$; or

- $T_{i}$ reads a version $w_{i}$ and $T_{k}$ commits version $w_{i}+1$.

Let $h^{\prime}$ be the completion of history $h$ such that a transaction $T_{k}$ in $h^{\prime}$ is committed in $h^{\prime}$ iff $T_{k}$ commits some version in $h$. Let $s$ be the following sequential TM history:

$$
s=h^{\prime}\left|T_{\sigma_{1}} \cdot h^{\prime}\right| T_{\sigma_{2}} \cdot \ldots,
$$

where $T_{\sigma_{1}} \ll T_{\sigma_{2}} \ll \ldots$ and $h^{\prime} \mid T_{\sigma_{j}}$ is the longest subsequence of $h^{\prime}$ consisting only of invocations and responses of $T_{\sigma_{j}}$. Clearly, $s$ is equivalent to $h^{\prime}$ and $s$ preserves the real-time order of $h$. Every transaction $T_{k}$ that is 
uses: $C$-compare-and-swap object; $\mathrm{R}[1, \ldots, \mathrm{n}]$-snapshot object (other variables are process-local) initially: $C=(1,(0,0, \ldots)), R[1, \ldots, n]=(0, \ldots, 0)$ version $=\perp$, timestamp $=0$, and count $=0$ (at every process $\left.p_{i}\right)$

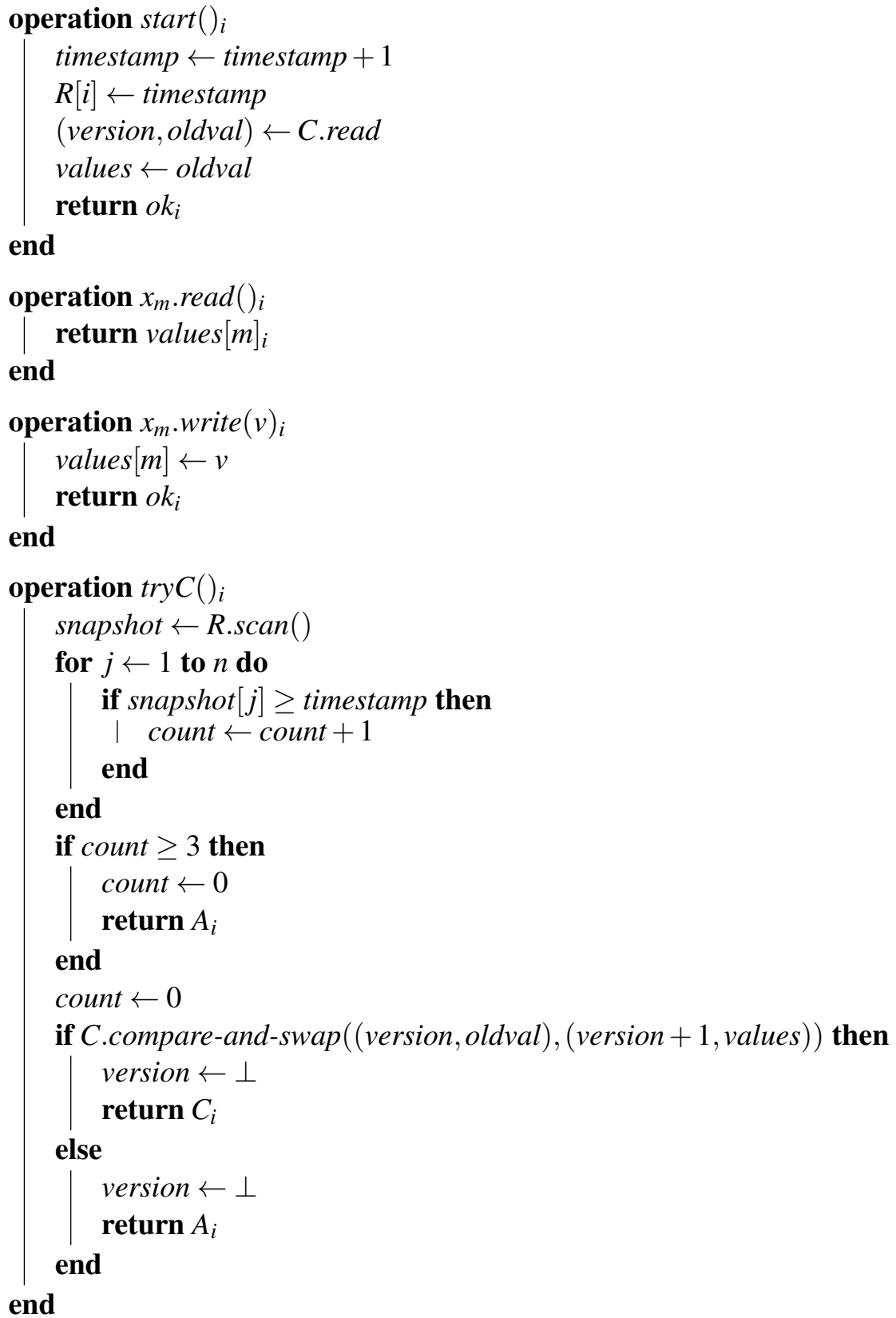

Algorithm 1: Algorithm $I_{(1,2)}$ implementing a TM that ensures $S$ and $(1,2)$-freedom. 
aborted in $s$ does not commit any version, and so $T_{k}$ does not change the state of base object $C$. Hence, aborted transactions are effectively invisible to other transactions. Every transaction $T_{k}$ that is committed in $s$ reads some version $w$ and commits version $w+1$. Since the version number stored in $C$ never decreases, no other transaction commits version $w+1$ in $h$. transaction $T_{k}$ thus reads the current snapshot of t-variable values from $C$, modifies the snapshot locally within operations read and write, and then atomically changes the state of $C$ from the old snapshot, with version number $w$, to the new snapshot, with version number $w+1$. Therefore, all transactions that follow $T_{k}$ in $s$ observe all the values written to t-variables by $T_{k}$, and by all preceding transactions that are committed in $s$. Therefore, history respects the sequential specification Seq of TM objects, and so history $h$ ensures opacity. This means that, TM implementation $I_{(1,2)}$ ensures opacity.

The second requirement of $S$. Observe that each time a process starts a new transaction it increments its timestamp by 1 and announces the new timestamp to other processes by updating the corresponding register $R[i]$. Let $h$ be any history of $I_{(1,2)}$ and let $T_{1}, T_{2}, T_{3}, \ldots$ be any three (or more) concurrent transactions in $h$, executed by different processes $p_{1}, p_{2}, p_{3}, \ldots$, respectively, s.t. there exists number $t$ s.t. for each $p_{i} \in$ $\left\{p_{1}, p_{2}, p_{3}, \ldots\right\}, T_{i}$ is the $t$-th transactions in $h \mid p_{i}$ and each $T_{i}$ invokes try $C_{i}$ after some other two transactions are returned a response for a start ()$_{j}$, where $j \neq i$ and $j \in\{1,2,3\}$, operation in $h$.

Because each $T_{1}, T_{2}, T_{3}, \ldots$ is the $t$-th transaction of a corresponding process, when $T_{1}, T_{2}, T_{3}, \ldots$ execute $\operatorname{start}()$, the timestamps of each process becomes $t$ and is written to the corresponding register. Since each $T_{1}, T_{2}, T_{3}$ invokes $\operatorname{try} C()$ after some other two processes get responses for $\operatorname{start}()$, each transaction observes the new timestamps of the other two transactions. Therefore, count becomes at least 3 during the execution of $\operatorname{try} C()$ of each of the transaction. And consequently, each of the transaction aborts in $h$.

$(1,2)$-Freedom. Consider a fair execution $e$ of $I_{(1,2)}$ and its corresponding history $h$ such that only two processes take infinitely many steps (and therefore only two processes are correct). Consider any correct and live, i.e. transaction which is not aborted or committed, transaction $T_{k}$ in history $h$. A transaction $T_{k}$ can be aborted by $I_{(1,2)}$ only if $T_{k}$ either it encounters that some other transaction has a higher timestamp tm or $T_{k}$ reads some version $w$ and then fails to commit version $w+1$. Since eventually there are only two processes that take steps, eventually transactions can be aborted only when $T_{k}$ reads some version $w$ and then fails to commit version $w+1$; this can happen only if some other transaction by the second correct process commits version $w+1$. Since a transaction that commits any version cannot be aborted, there are infinitely many transactions in $h$ that are not aborted, and so history $h$, and thus also TM implementation $I_{(1,2)}$, ensures $(1,2)$-freedom.

\section{Concluding Remarks}

Our impossibility results mean that if we consider too general definitions of liveness, then it is impossible to answer questions about strongest (weakest) implementable (non-implementable) liveness property. To circumvent these impossibility results it is necessary to consider more restricted definitions of liveness. We have considered one such restriction in the previous section. Here we discuss alternative ones.

For example, we may consider the notion of $S$-freedom [36], which states that for every set of correct processes $P$, where $S$ is a set containing some natural numbers and $|P| \in S$, every process in $P$ should make progress as long as it does not encounter step contention with processes outside $P$. A characterization of liveness properties of consensus objects is given in [36] which partitions liveness properties into implementable (using registers only) and non-implementable properties; $S$-freedom is then shown to be implementable iff $|S|=1$. However, even such restricted definition of liveness does not allow to determine a strongest (or a weakest) implementable (or non-implementable) liveness property with a safety property like consensus agreement and validity. Because none of the $S$-freedom properties with $|S|=1$ is comparable to each other, there is no strongest $S$-freedom consensus liveness property implementable using only registers. 
One way to circumvent the issue is to consider a restricted definition of liveness which totally orders all liveness properties. For example, the notion of $(n, x)$-liveness [25] states that $x$ processes should be waitfree and $n-x$ processes should be obstruction-free. It is shown [25] that if $x \geq 1$, then it is impossible to implement a consensus object from registers. Since the set of all $(n, x)$-liveness properties is totally ordered, then the strongest (and the only) implementable liveness property is $(n, 0)$-liveness, and the weakest nonimplementable liveness property is $(n, 1)$-liveness. Liveness properties can also be defined based on the notion of $k$-obstruction-freedom [35] which states that if processes from a set $P$, where $|P| \leq k$, do not encounter step contention with processes outside of $P$, then every process from $P$ should make progress. According to this definition, the strongest implementable liveness property of a consensus object is 1-obstruction-freedom and the weakest non-implementable is 2-obstruction-freedom. However, the definitions of liveness in [25, 35] do not include some common liveness properties, e.g. lock-freedom that requires progress for at least one process. There is a compromise between how general a definition of liveness should be and the possibility of totally ordering the defined liveness properties.

\section{Acknowledgements}

We are very grateful to Cheng Wang who discovered the mistake on the example of an adversary set for consensus. We also wish to thank the anonymous reviewers for their helpful comments.

This work has been supported by the European Commission under the 7th Framework Program through the TransForm (FP7-MC-ITN-238639) project and by the European Research Council under the AdversaryOriented Computing project (ERC-2013-AdG-339539-AOC). 


\section{References}

[1] B. Alpern and F. B. Schneider. Defining liveness. Information Processing Letters, 21(4), 1985.

[2] H. Attiya and J. Welch. Distributed Computing: Fundamentals, Simulations and Advanced Topics. John Wiley \& Sons, 2004.

[3] E. Borowsky and E. Gafni. Generalized flp impossibility result for t-resilient asynchronous computations. In ACM STOC, 1993.

[4] V. Bushkov, R. Guerraoui, and M. Kapałka. On the liveness of transactional memory. In ACM PODC, 2012.

[5] B. Chor, A. Israeli, and M. Li. On processor coordination using asynchronous hardware. In ACM PODC, 1987.

[6] F. Ellen, P. Fatourou, E. Kosmas, A. Milani, and C. Travers. Universal constructions that ensure disjoint-access parallelism and wait-freedom. In ACM PODC, 2012.

[7] F. Fich and E. Ruppert. Hundreds of impossibility results for distributed computing. Distrib. Comput., 16(2-3), 2003.

[8] M. J. Fischer, N. A. Lynch, and M. S. Paterson. Impossibility of distributed consensus with one faulty process. J. ACM, 32(2), 1985.

[9] K. Fraser. Practical lock freedom. In PhD thesis, Cambridge University Computer Laboratory, 2003.

[10] P. Ganty and R. Majumdar. Algorithmic verification of asynchronous programs. ACM Trans. Program. Lang. Syst., $34(1), 2012$.

[11] S. Gilbert and N. Lynch. Brewer's conjecture and the feasibility of consistent, available, partition-tolerant web services. SIGACT News, 33(2), 2002.

[12] S. Gilbert and N. A. Lynch. Perspectives on the cap theorem. Computer, 45(2), 2012.

[13] D. S. Greenberg, G. Taubenfeld, and D.-W. Wang. Choice coordination with multiple alternatives (preliminary version). In WDAG, 1992.

[14] R. Guerraoui and M. Kapalka. On obstruction-free transactions. In ACM SPAA, 2008.

[15] R. Guerraoui and M. Kapalka. On the correctness of transactional memory. In ACM PPoPP, 2008.

[16] R. Guerraoui and M. Kapalka. Principles of Transactional Memory. Morgan and Claypool, 2010.

[17] R. Guerraoui and E. Ruppert. Anonymous and fault-tolerant shared-memory computing. Distributed Computing, 20(3):165$177,2007$.

[18] T. Harris, J. R. Larus, and R. Rajwar. Transactional Memory, 2nd edition. Morgan and Claypool, 2010.

[19] M. Herlihy. Wait-free synchronization. ACM Trans. Program. Lang. Syst., 13(1), 1991.

[20] M. Herlihy, V. Luchangco, and M. Moir. Obstruction-free synchronization: Double-ended queues as an example. In IEEE ICDCS, 2003.

[21] M. Herlihy, V. Luchangco, M. Moir, and W. N. Scherer, III. Software transactional memory for dynamic-sized data structures. In $A C M$ PODC, 2003.

[22] M. Herlihy and N. Shavit. The topological structure of asynchronous computability. J. ACM, 46(6), 1999.

[23] M. Herlihy and N. Shavit. On the nature of progress. In OPODIS, 2011.

[24] M. P. Herlihy and J. M. Wing. Linearizability: a correctness condition for concurrent objects. ACM Trans. Program. Lang. Syst., 12(3), 1990.

[25] D. Imbs, M. Raynal, and G. Taubenfeld. On asymmetric progress conditions. In ACM PODC, 2010.

[26] L. Lamport. Using time instead of timeout for fault-tolerant distributed systems. ACM Trans. Program. Lang. Syst., 6(2), 1984.

[27] B. W. Lampson. How to build a highly available system using consensus. In WDAG, 1996.

[28] M. C. Loui and H. H. Abu-Amara. Memory requirements for agreement among unreliable asynchronous processes, volume 4. JAI press, 1987. 
[29] N. A. Lynch. Distributed Algorithms. Morgan Kaufmann Publishers Inc., 1996.

[30] S. Owicki and L. Lamport. Proving liveness properties of concurrent programs. ACM Trans. Program. Lang. Syst., 4(3), 1982.

[31] C. H. Papadimitriou. The serializability of concurrent database updates. J. ACM, 26(4), 1979.

[32] F. B. Schneider. Implementing fault-tolerant services using the state machine approach: a tutorial. ACM Comput. Surv., 22(4), 1990.

[33] R. Segala, R. Gawlick, J. Søgaard-Andersen, and N. Lynch. Liveness in timed and untimed systems. Inf. Comput., 141(2), 1998.

[34] N. Shavit and D. Touitou. Software transactional memory. In ACM PODC, 1995.

[35] G. Taubenfeld. On the computational power of shared objects. In OPODIS, 2009.

[36] G. Taubenfeld. The computational structure of progress conditions. In DISC, 2010.

[37] G. Taubenfeld and S. Moran. Possibility and impossibility results in a shared memory environment. Acta Inf, 33, 1996.

[38] H. Völzer and D. Varacca. Defining fairness in reactive and concurrent systems. J. ACM, 59(3), 2012. 